\title{
Discovery of a tidal disruption event candidate from the 2XMM catalog
}

\author{
D. Lin ${ }^{1,2,3, a}$, E.R. Carrasco ${ }^{4}$, D. Grupe ${ }^{5}$, N.A. Webb ${ }^{1,2}$, D. Barret ${ }^{1,2}$, and S.A. Farrell ${ }^{6}$ \\ ${ }^{1}$ CNRS, IRAP, 9 avenue du Colonel Roche, BP. 44346, 31028 Toulouse Cedex 4, France \\ 2 Université de Toulouse, UPS-OMP, IRAP, Toulouse, France \\ ${ }^{3}$ Department of Physics and Astronomy, University of Alabama, Tuscaloosa, AL 35487, USA \\ ${ }^{4}$ Gemini ObservatoryIAURA, Southern Operations Center, Casilla 603, La Serena, Chile \\ 5 Department of Astronomy and Astrophysics, Pennsylvania State University, 525 Davey Lab, University Park, \\ PA 16802, USA \\ ${ }^{6}$ Sydney Institute for Astronomy (SIfA), School of Physics, The University of Sydney, NSW 2006, Australia
}

\begin{abstract}
Stars approaching a supermassive black hole (SMBH) can be tidally disrupted and subsequently accreted, providing a unique way to find and study inactive SMBHs. We report on our discovery of a new tidal disruption event candidate, 2XMMi J184725.1-631724, with unprecedented ultrasoft X-ray spectra near the flare peak. It lies toward the center of an inactive galaxy at $z=0.0353$. It was detected serendipitously in two XMM-Newton observations separated by 211 days, with the flux increasing by a factor of $\sim 9$. The source was not detected in X-rays by ROSAT in 1992, indicating a long-term variability factor of $>64$; neither by Swift in 2011, implying a flux decay factor of $>12$ since the last XMM-Newton observation. The XMM-Newton $\mathrm{X}$-ray spectra are dominated by a strong cool thermal disk $(>80 \%$, tens of $\mathrm{eV}$ ) with the luminosity appearing to follow the $L \propto T^{4}$ relation, often seen in the thermal state of the BH X-ray binaries. Both XMM-Newton observations show large variability on timescales of hours. This can be explained as due to fast variations in the mass accretion rate, maybe caused by the shocks during the tidal disruption of the star.
\end{abstract}

\section{INTRODUCTION}

Supermassive black holes (SMBHs) are believed to reside in the center of many galaxies, but only a fraction of them reveal themselves as active galactic nuclei (AGN), while others are dormant [1]. Tidal disruption events (TDEs) can provide a unique way to find and study the dormant SMBHs [2,3]. Such an event occurs when a star approaches a SMBH and is tidally disrupted and subsequently accreted [2,3]. TDEs are expected to be very rare, with the average occurrence rate of $\sim 10^{-4} \mathrm{yr}^{-1}$ per galaxy [4], and only about a dozen of TDE candidates have been found (e.g., [5-12]), since the ROSAT All-Sky Survey two decades ago.

TDEs are predicted to share the following properties if the disrupted star is solar-type [2-4,13,14]: (1) being transient, with a fast rise on a timescale of months, and the decay can last for months to years, with the luminosity decaying as $L \propto\left(t-t_{\mathrm{D}}\right)^{-5 / 3}\left(t_{\mathrm{D}}\right.$ is the tidal disruption time); (2) peak luminosity reaching the Eddington limit $\left(\sim 10^{44} \mathrm{erg} \mathrm{s}^{-1}\right.$ for a $\left.10^{6}-M_{\odot} \mathrm{SMBH}\right)$; (3) coincidence with the center of a galaxy which shows no sign of nuclear activity history; and (4) dominant thermal UV or X-ray emission, with characteristic temperatures $<0.1 \mathrm{keV}$. Most TDE candidates more or less show the above properties. Swift J164449.3+573451 and Swift J2058.4+0516 are two exceptions, which are hard X-ray transients and are probably due to observation of a relativistic jet pointed toward us [15-17].

During our project of classification of a sample of sources from the 2XMMi-DR3 Catalog [18], we discovered the ultrasoft X-ray transient 2XMMi J184725.1631724 (XMMJ1847 hereafter, [19]). Here we show that

a e-mail: dlin@ua.edu this source has the TDE characteristics listed above and thus is a strong TDE candidate. Moreover, it has the best X-ray spectra with dominant thermal emission ever obtained for TDE candidates, allowing more details of such events to be revealed [19].

\section{XMMJ1847 AS A TIDAL DISRUPTION EVENT CANDIDATE}

Figure 1 shows the four X-ray pointed observations which covered XMMJ1847. The source was serendipitously detected in two XMM-Newton observations (XMM1 and XMM2 hereafter) in 2006-2007, which separated by 211 days, with the $0.2-10 \mathrm{keV}$ absorbed flux increasing by a factor of $\sim 9$ [19]. However, it was not detected in a deep ROSAT observation in 1992, indicating a flux lower than XMM2 by a factor of $>64$ [19]. At our request, Swift made an observation of XMMJ1847 for 5 ks and the source was barely detected, indicating that the $0.2-10 \mathrm{keV}$ flux had decreased by a factor of $>12$ since XMM2. Figure 2 plots the long-term luminosity curve. Two decay curves following $L \propto\left(t-t_{\mathrm{D}}\right)^{-5 / 3}$ are also plotted, assuming XMM2 in the decay phase and two reasonable values of $t_{\mathrm{D}}$ : the dotted and solid curves for $t_{\mathrm{D}}$ at half a year and one month before XMM1, respectively. These curves predict that the source luminosity in the Swift observation should be a factor of about 20 lower than XMM2, and the detection limit obtained is consistent with this. Thus XMMJ1847 has the transient nature and long-term evolution expected for a TDE [19]. The peak luminosity reached near $10^{44} \mathrm{erg} \mathrm{s}^{-1}$, as seen in most other TDE candidates.

XMMJ1847 is coincident with the center of the galaxy IC 4765-f01-1504, as shown in Figure 3, which plots the 

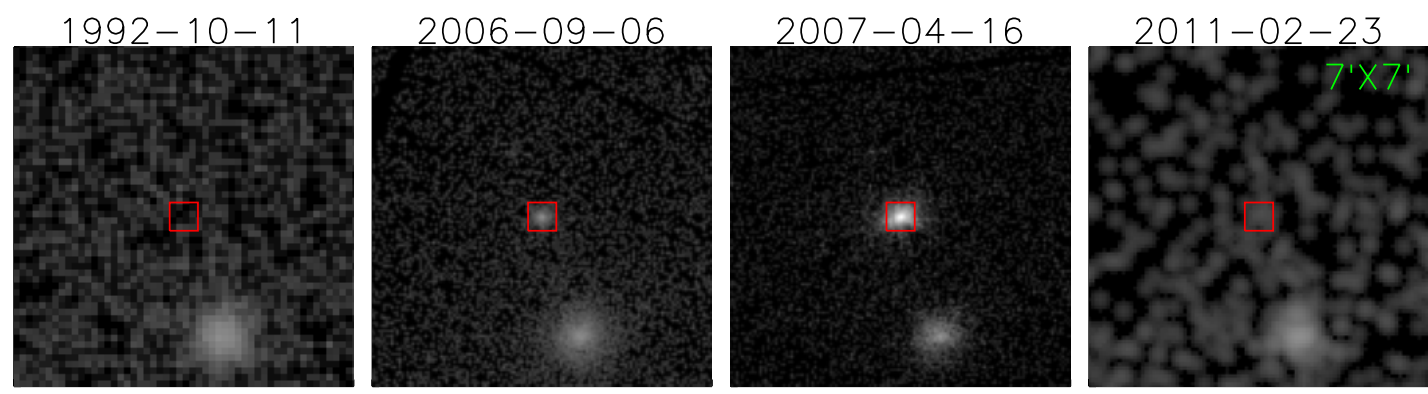

Figure 1. The count images of 2XMMi J184725.1-631724 (red box) from four X-ray observations. The first one is from ROSAT, the middle two are from XMM-Newton/MOS1 and the last one is from Swift/XRT. The bright extended source at the bottom of each image is from the giant cD elliptical IC 4765, which is expected to be steady and can be used as reference for comparison.

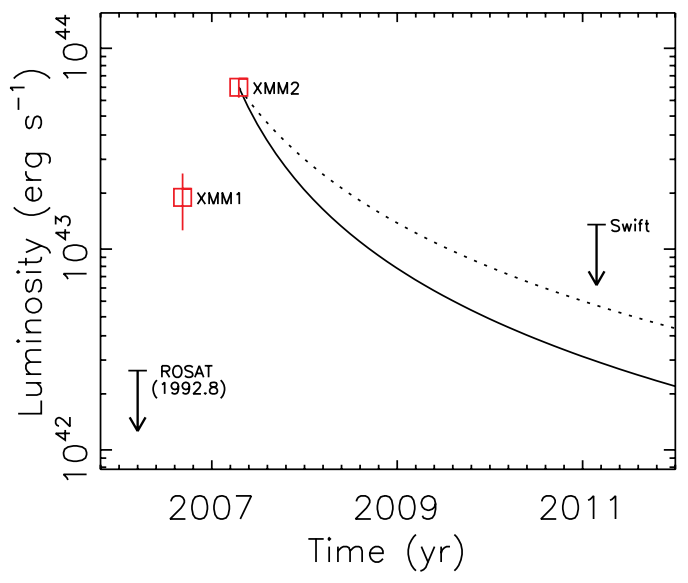

Figure 2. The long-term luminosity curve inferred from X-ray spectral fits [19]. Arrows represent 3- $\sigma$ upper bounds. Note that the ROSAT observation was made in 1992 October. The solid curve is Luminosity $=3.45 \times$ $10^{43}[(\text { Time }-2006.60 \mathrm{yr}) /(1 \mathrm{yr})]^{-5 / 3} \mathrm{erg} \mathrm{s}^{-1}$, and the dotted curve is Luminosity $=7.57 \times 10^{43}[(\text { Time }-2006.18 \mathrm{yr}) /(1 \mathrm{yr})]^{-5 / 3} \mathrm{erg} \mathrm{s}^{-1}$.

V-filter image obtained by the $1.3 \mathrm{~m}$ Warsaw telescope at Las Campanas Observatory in Chile [20]. The Gemini South Telescope took an optical spectrum of the galaxy on 2011 March 19 (about four years after XMM2), which shows only typical absorption features at a redshift of $z=0.0353$ (Figure 4). There are no clear emission lines detected, indicating the lack of strong nuclear activity of the galaxy [19]. Assuming a flat universe with $H_{0}=$ $73 \mathrm{~km} \mathrm{~s}^{-1} \mathrm{Mpc}^{-1}$ and $\Omega_{\mathrm{M}}=0.27$, the above redshift corresponds to a luminosity distance of $149 \mathrm{Mpc}$.

The extreme softness of the X-ray spectra of XMMJ1847 can be seen in Figure 5, which shows the unfolded spectra of the two XMM-Newton observations fitted with a multicolor disk (MCD) plus a power-law (PL). Both spectra are dominated by the thermal disk emission with disk temperature $<0.1 \mathrm{keV}$ (Table 1). The disk luminosity is consistent with $L \propto k T_{\mathrm{MCD}}^{4}$, meaning that the inner disk radius is consistent with being constant [19]. These properties are very similar to the thermal state of stellar-mass black hole (BH) X-ray binaries [21], except that the disk is much cooler in XMMJ1847, which is expected if the accreting black hole in it is super massive [22].

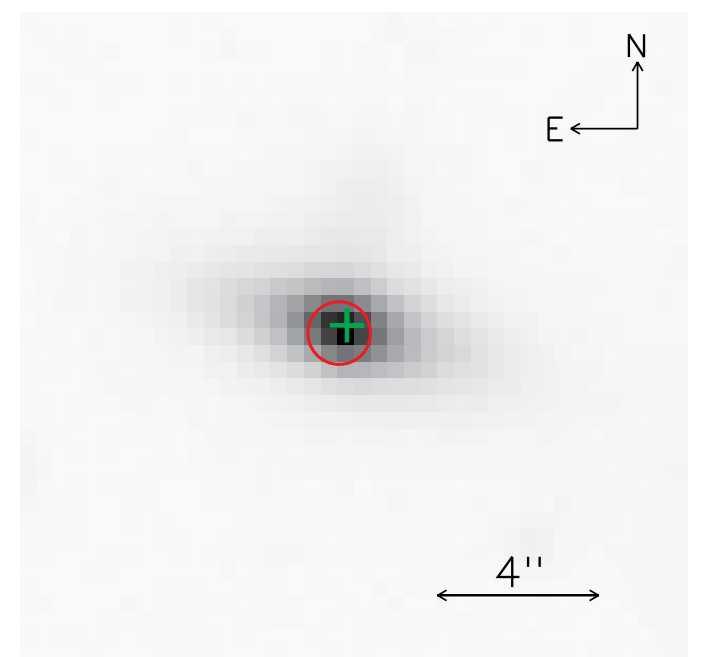

Figure 3. The optical image of the galaxy in the $\mathrm{V}$ band [19]. The pixel size is 0.414 . The green plus marks the central position of the galaxy obtained from the fits to its V- and Iband profiles using a Sérsic model, and it is at $\mathrm{RA}=18: 47: 25.14$, Dec=-63:17:24.77 (J2000). The red circle is centered at the X-ray position (RA=18:47:25.16, Dec $=-63: 17: 24.96(\mathrm{~J} 2000)$ ), with the radius corresponding to the $3-\sigma$ error of $0 .{ }^{\prime} 8$.

The source exhibited strong fast variability in both XMM-Newton observations, as shown in Figure 6 [19]. The left panels plot the pn $250 \mathrm{~s}$ background-subtracted light curves, and the right panels plot the power density spectra (PDS). For XMM2, which has higher count rates, we see that the source varies by a factor of $\sim 4$ within $5 \mathrm{ks}$. The PDS are flat at frequencies above $0.1 \mathrm{~Hz}$, indicating that the Poisson level (the black solid line) is reached. However, below $0.01 \mathrm{~Hz}$, both PDS show a clear deviation from the Poisson level. The fractional rms values within 0.0001 to $0.01 \mathrm{~Hz}$ after subtracting the Poisson level are $(21.2 \pm 5.3) \%$ and $(21.1 \pm 1.9) \%$ for XMM1 and XMM2, respectively. They are much larger than values of $\lesssim 5 \%$ typically seen in the thermal state of neutron-star or BH low-mass X-ray binaries [21,23,24]. To understand the cause of the strong fast variability of XMMJ1847, we fit spectra extracted from the high- $\left(>0.8\right.$ counts s$\left.^{-1}\right)$ and lowcount-rate periods of the bright observation XMM2 and find that the MCD temperature in the low state is smaller than in the high state by $10 \mathrm{eV}$ at a 4.4- $\sigma$ confidence level, while the MCD normalizations (thus the inner disk radius) 


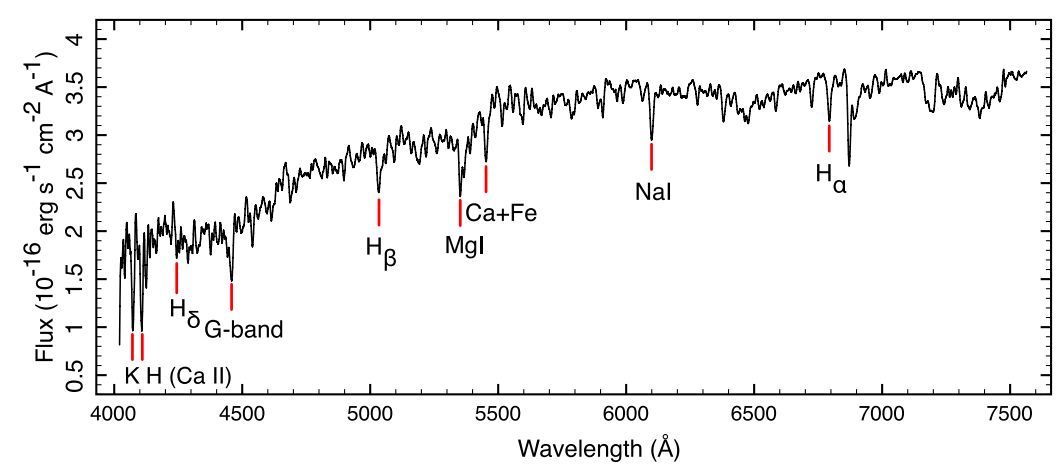

Figure 4. The smoothed spectrum of the galaxy IC 4765-f01-1504 from the Gemini South Telescope, with the most important absorption lines identified [19]. The drop at $6870 \AA$ is due to the atmosphere $\mathrm{OH}$ absorption.
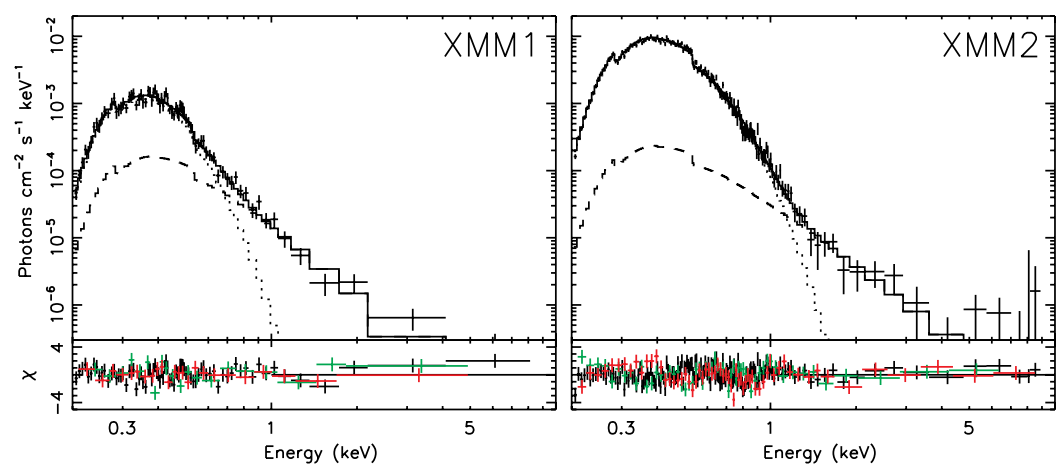

Figure 5. The unfolded spectra and the fit residuals using the model MCD+PL [19]. For clarity, only the pn spectra are shown for the unfolded spectra. The dotted, dashed, and solid lines are for the MCD and PL components and the total model, respectively. The residuals are shown for all three cameras (black/red/green for pn/MOS1/MOS2, respectively).

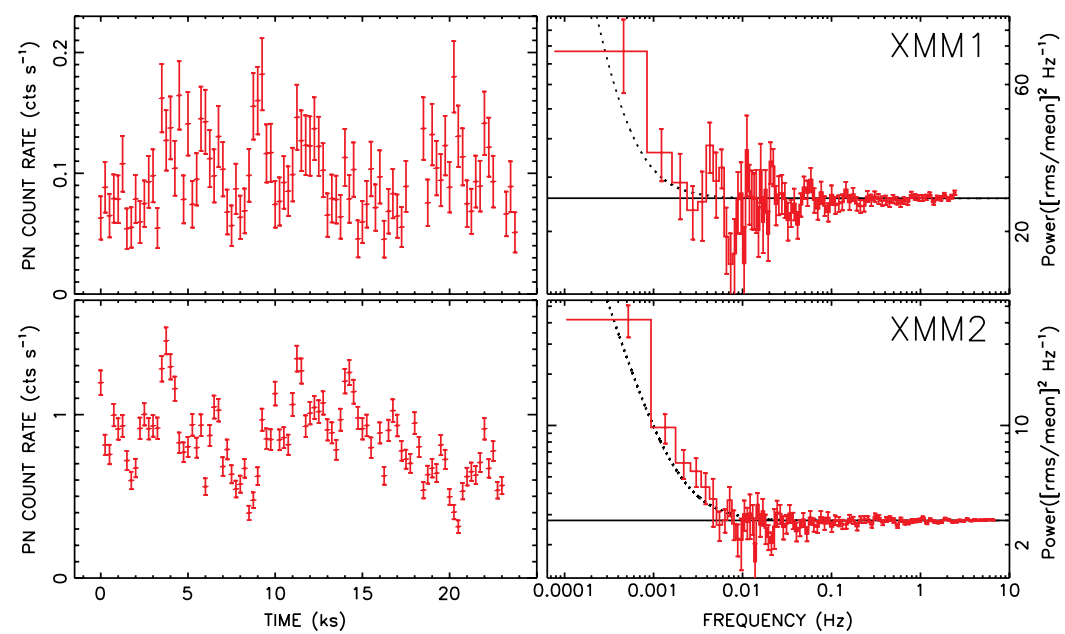

Figure 6. Left panels: The pn $250 \mathrm{~s} 0.2-2.0 \mathrm{keV}$ background-subtracted light curves. Right panels: The PDS of the pn $0.2-2.0 \mathrm{keV}$ light curves binned at $199.1 \mathrm{~ms}$ for XMM1 and $73.4 \mathrm{~ms}$ for XMM2. The black solid constant line is the average PDS above $0.1 \mathrm{~Hz}$, representing the Poisson level, and the dotted line is the best-fitting model of a PL plus a constant [19].

are consistent with being the same within the error bars. Thus the large fast variability of XMMJ1847 could be due to the fast variations in the mass accretion rate, possibly caused by shocks during tidal disruption of the star and/or circularization of the disrupted material into the accretion disk. This is not seen in BH X-ray binaries in the thermal state and may be intrinsic to TDEs. Although the TDE candidate Swift J164449.3+573451 also showed large fast variability, its origin is not so clear and can be produced in the jet [15] or due to fast variations in the mass accretion rate as above [25]. The fast variability is seldom studied for other TDE candidates, due to their lack of X-ray data of high quality.

The BH mass can be estimated in several ways: using the $\mathrm{BH}$ mass vs. bulge luminosity relations; fitting the $\mathrm{X}$-ray spectra with a relativistic accretion disk model such 
Table 1. Spectral modeling results [19]. The column $f_{\mathrm{MCD} / \mathrm{BB}}$ refers to the unabsorbed flux fraction of the MCD or blackbody (BB) component in the $0.2-10 \mathrm{keV}$ energy band. $F_{\text {abs }}$ and $F_{\text {unabs }}$ are the total absorbed and unabsorbed fluxes in the $0.2-10 \mathrm{keV}$ energy band, respectively. The luminosity $L$ was calculated using the unabsorbed bolometric flux of each spectral component (the PL component was integrated down to $0.2 \mathrm{keV}$ ). All errors are at a $90 \%$-confidence level.

\begin{tabular}{|c|c|c|c|c|c|c|c|c|c|c|}
\hline Model & Obs & $\begin{array}{c}N_{\mathrm{H}} \\
\left(10^{20} \mathrm{~cm}^{-2}\right)\end{array}$ & $\begin{array}{c}k T_{\mathrm{MCD} / \mathrm{BB}} \\
(\mathrm{eV})\end{array}$ & $\begin{array}{c}N_{\mathrm{MCD} / \mathrm{BB}} \\
\left(10^{4}\right)\end{array}$ & $\Gamma_{\mathrm{PL}}$ & $\begin{array}{c}N_{\mathrm{PL}} \\
\left(10^{-5} / \%\right)\end{array}$ & $\chi_{v}^{2}(v)$ & $\begin{array}{c}f_{\mathrm{MCD} / \mathrm{BB}} \\
(\%)\end{array}$ & $\begin{array}{cr}F_{\text {abs }} & F_{\text {unabs }} \\
\left(10^{-12} \mathrm{erg} \mathrm{s}^{-1} \mathrm{~cm}^{-2}\right)\end{array}$ & $\begin{array}{c}L \\
\left(10^{43} \mathrm{erg} \mathrm{s}^{-1}\right)\end{array}$ \\
\hline \multirow[t]{2}{*}{$\mathrm{MCD}+\mathrm{PL}$} & XMM1 & $8.6 \pm 0.5$ & $65.8 \pm 5.0$ & $1.56_{-0.63}^{+1.21}$ & $3.72 \pm 0.62$ & $1.79 \pm 0.32$ & $0.92(115)$ & $84.5_{-14.8}^{+7.3}$ & $0.22 \pm 0.01 \quad 1.76 \pm 0.19$ & $1.70 \pm 0.44$ \\
\hline & XMM2 & & $93.1 \pm 2.2$ & $1.45_{-0.23}^{+0.34}$ & $3.27 \pm 0.70$ & $3.75 \pm 1.39$ & $1.20(284)$ & $96.4_{-6.1}^{+1.9}$ & $1.93 \pm 0.03 \quad 10.18_{-0.57}^{+1.00}$ & $6.38 \pm 0.66$ \\
\hline \multirow[t]{2}{*}{$\mathrm{BB}+\mathrm{PL}$} & XMM1 & $7.4 \pm 0.7$ & $57.8 \pm 3.9$ & $1.63_{-0.56}^{+1.13}$ & $3.71 \pm 0.59$ & $1.76 \pm 0.28$ & $0.92(115)$ & $78.2_{-17.2}^{+9.5}$ & $0.22 \pm 0.01 \quad 1.23 \pm 0.11$ & $0.59 \pm 0.14$ \\
\hline & XMM2 & & $78.2 \pm 1.6$ & $2.37 \pm 0.41$ & $3.89 \pm 0.61$ & $5.54 \pm 1.46$ & $1.18(284)$ & $86.0_{-14.4}^{+7.6}$ & $1.92 \pm 0.03 \quad 7.59_{-0.51}^{+1.15}$ & $2.79 \pm 0.39$ \\
\hline
\end{tabular}

as kerrbb in XSPEC; assuming the timescale on which the source begins to show strong variability $(\sim 100 \mathrm{~s}$, Figure 6$)$ as the light crossing time of the inner accretion disk around a $\mathrm{BH}[19]$. They all infer a $\mathrm{BH}$ of about $10^{5}-10^{6} M_{\odot}$. Thus the peak luminosity (based on XMM2) reached about the Eddington limit, as expected for a TDE.

\section{DISCUSSION AND CONCLUSIONS}

We have demostrated that XMMJ1847 has many properties expected for a TDE: it is an ultrasoft X-ray transient coincident with the center of a galaxy $(z=0.0353)$ that shows no obvious nuclear activity. The peak luminosity reaches the Eddington limit for a $\mathrm{BH}$ of $10^{5}-10^{6} \mathrm{M}_{\odot}$. With data of high quality, we find that the source behaved similar to the thermal state of stellar-mass BH X-ray binaries in that the two XMM-Newton spectra are dominated by a thermal disk component with luminosity $L \propto k T^{4}$. Strong fast variability on timescales of hours was also seen in both observations and could be explained as due to fast variations in the mass accretion rate, maybe caused by the shocks during the tidal disruption of the star. Compared with the high quality of data near the flare peak, its decay still needs to be covered by future observations.

Other explanations for XMMJ1847 can be ruled out as follows. Assuming that the source is in the galaxy IC 4765-f01-1504, then it is most likely an ultraluminous offnuclear X-ray source, but this explanation is not attractive considering that the source is within $0.5 \mathrm{kpc}(3 \sigma$ positional error) from the center of the galaxy and that its spectral and timing properties infer that the $\mathrm{BH}$ is supermassive. It is unlikely to be a foreground star either because its $\mathrm{X}$-ray-to-IR flux ratio $\left(\log \left(F_{\mathrm{X}} / F_{\mathrm{IR}}\right)=0.86\right)$ is much larger than those of stars $\left(\log \left(F_{\mathrm{X}} / F_{\mathrm{IR}}\right) \lesssim-0.9,[18]\right)$. Finally, it is unlikely to be a Galactic supersoft X-ray source, because such sources are rare and the probability for one of them to be coincident with the center of a nearby galaxy is essentially zero [19].

\section{References}

[1] J. Kormendy, D. Richstone, ARA\&A 33, 581 (1995)

[2] V.V. Lidskii, L.M. Ozernoi, Soviet Astronomy Letters 5, 16 (1979)

[3] M.J. Rees, Nature 333, 523 (1988)
[4] M.J. Rees, Science 247, 817 (1990)

[5] D. Grupe, H. Thomas, K.M. Leighly, A\&A 350, L31 (1999)

[6] S. Komossa, J. Greiner, A\&A 349, L45 (1999)

[7] S. Komossa, N. Bade, A\&A 343, 775 (1999)

[8] P. Esquej, R.D. Saxton, M.J. Freyberg, A.M. Read, B. Altieri, M. Sanchez-Portal, G. Hasinger, A\&A 462, L49 (2007)

[9] P. Esquej, R.D. Saxton, S. Komossa, A.M. Read, M.J. Freyberg, G. Hasinger, D.A. García-Hernández, H. Lu, J. Rodriguez Zaurín, M. Sánchez-Portal et al., A\&A 489, 543 (2008)

[10] S. Gezari, T. Heckman, S.B. Cenko, M. Eracleous, K. Forster, T.S. Gonçalves, D.C. Martin, P. Morrissey, S.G. Neff, M. Seibert et al., ApJ 698, 1367 (2009)

[11] W.P. Maksym, M.P. Ulmer, M. Eracleous, ApJ 722, 1035 (2010)

[12] R.D. Saxton, A.M. Read, P. Esquej, S. Komossa, S. Dougherty, P. Rodriguez-Pascual, D. Barrado, A\&A 541, A106 (2012)

[13] S. Komossa, Ludwig Biermann Award Lecture: Xray Evidence for Supermassive Black Holes at the Centers of Nearby, Non-Active Galaxies, in Reviews in Modern Astronomy, edited by R. E. Schielicke (2002), Vol. 15 of Reviews in Modern Astronomy, pp. 27-+

[14] S. Komossa, in The Universe in X-rays (eds. J. E. Trümper \& G. Hasinger, Springer, 2008), pp. 367-371

[15] D.N. Burrows, J.A. Kennea, G. Ghisellini, V. Mangano, B. Zhang, K.L. Page, M. Eracleous, P. Romano, T. Sakamoto, A.D. Falcone et al., Nature 476, 421 (2011)

[16] B.A. Zauderer, E. Berger, A.M. Soderberg, A. Loeb, R. Narayan, D.A. Frail, G.R. Petitpas, A. Brunthaler, R. Chornock, J.M. Carpenter et al., Nature 476, 425 (2011)

[17] S.B. Cenko, H.A. Krimm, A. Horesh, A. Rau, D.A. Frail, J.A. Kennea, A.J. Levan, S.T. Holland, N.R. Butler, R.M. Quimby et al., ApJ 753, 77 (2012)

[18] D. Lin, N.A. Webb, D. Barret, ApJ 756, 27 (2012)

[19] D. Lin, E.R. Carrasco, D. Grupe, N.A. Webb, D. Barret, S.A. Farrell, ApJ 738, 52 (2011)

[20] E.R. Carrasco, C. Mendes de Oliveira, L. Infante, AJ 132, 1796 (2006) 
[21] R.A. Remillard, J.E. McClintock, ARA\&A 44, 49 (2006)

[22] M. Gierliński, C. Done, MNRAS 349, L7 (2004), arXiv: astro-ph/0312271

[23] D. Lin, R.A. Remillard, J. Homan, ApJ 667, 1073 (2007)
[24] D. Lin, R.A. Remillard, J. Homan, ApJ 696, 1257 (2009)

[25] J.S. Bloom, D. Giannios, B.D. Metzger, S.B. Cenko, D.A. Perley, N.R. Butler, N.R. Tanvir, A.J. Levan, P.T. O'Brien, L.E. Strubbe et al., Science 333, 203 (2011) 